\title{
Subcutaneous delivery of sumatriptan in the treatment of migraine and primary headache
}

REVIEW

This article was published in the following Dove Press journal:

Patient Preference and Adherence

30 December 201I

Number of times this article has been viewed

\section{Johanna C Moore \\ James R Miner}

Department of Emergency Medicine, Hennepin County Medical Center, Minneapolis, MN, USA

Video abstract

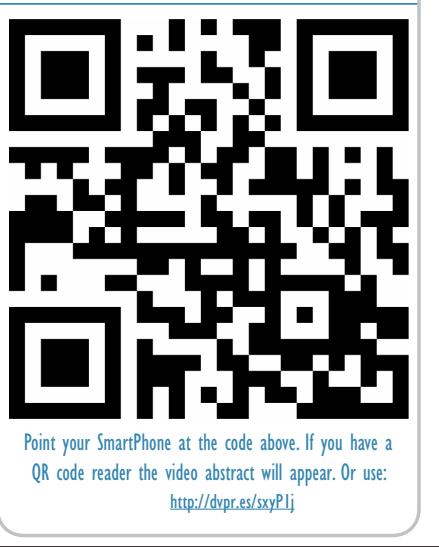

Correspondence: James R Miner Department of Emergency Medicine, Hennepin County Medical Center, 70I Park Ave, Minneapolis,

MN 554I5, USA

$\mathrm{Tel}+\mathrm{I} 6128735683$

Fax +l 6129044242

Emailminer0I5@umn.edu
Abstract: Subcutaneous sumatriptan is an effective treatment for pain from acute migraine headache, and can be used in patients with known migraine syndrome and in patients with primary headaches when secondary causes have been excluded. In limited comparative trials, subcutaneous sumatriptan performed in a manner comparable with oral eletriptan and intravenous metoclopramide, was superior to intravenous aspirin and intramuscular trimethobenzamidediphenhydramine, and was inferior to intravenous prochlorperazine for pain relief. The most common side effects seen with subcutaneous sumatriptan are injection site reactions and triptan sensations. As with all triptans, there is a risk of rare cardiovascular events with subcutaneous sumatriptan and its use should be limited to those without known cerebrovascular disease and limited in those with known cardiovascular risk factors and unknown disease status. In studies of patient preference and tolerability, the subcutaneous formulation has a faster time of onset and high rate of efficacy when compared with the oral formulation, but the oral formulation appears to be better tolerated. It is important to consider the needs of the patient, their past medical history, and what aspects of migraine treatment are most important to the patient when considering treatment of acute migraine or primary headache. Subcutaneous sumatriptan is a good first-line agent for the treatment of pain from acute migraine headaches and primary headaches.

Keywords: sumatriptan, subcutaneous, migraine headache, primary headache

\section{Introduction}

Sumatriptan belongs to a class of medications known as the triptans. Sumatriptan, the first of these medications, was introduced in the early 1990s and revolutionized the current treatment of migraine. Like other triptans, sumatriptan is an agonist at the serotonin $5 \mathrm{HT}_{1 \mathrm{~B}}$ and $5 \mathrm{HT}_{1 \mathrm{D}}$ receptor subtypes. Sumatriptan has demonstrated efficacy when administered by the subcutaneous, oral, intranasal, or rectal route.

Although sumatriptan is primarily used and studied in the setting of migraine headache, it is also an effective agent for cluster headaches. ${ }^{1}$ The recent literature has also shown that sumatriptan can be safely and effectively used in patients with primary headache, ie, headaches that are not secondary to a discrete pathology, including migraine, probable migraine, tension, and cluster headaches. ${ }^{2}$ This review will focus on the efficacy, safety, and patient acceptability and compliance concerning subcutaneous administration of sumatriptan for the treatment of migraine and primary headache.

The exact mechanism of action of triptans is unclear. Initially they were thought to promote vasoconstriction of cerebral vessels that are abnormally vasodilated during migraine headaches and possibly inhibit areas of neurogenic inflammation around these blood vessels. ${ }^{3}$ However, sumatriptan likely also acts by reducing neuronal activity 
within the trigeminovascular system..$^{4-7}$ Further, sumatriptan may block the release of vasoactive neuropeptides from both peripheral and central trigeminal neurons by binding to $5-\mathrm{HT}_{1 \mathrm{~B} / \mathrm{D}}$ receptors presynaptically, preventing central sensitization and therefore migraine-associated hyperalgesia. ${ }^{8}$ It has also been shown that triptans modulate transmission of trigeminovascular neurons at the level of the thalamus ${ }^{4}$ and periaqueductal gray matter, ${ }^{9}$ suggesting that triptans may act at a higher level of the brain than was previously thought to regulate painful sensory information. ${ }^{4}$

\section{Efficacy}

All triptans have been shown to be superior to placebo for the treatment of acute migraine headaches. Of the varied delivery systems, subcutaneous sumatriptan has been shown in multiple studies to have the fastest onset of pain relief. ${ }^{3}$

Subcutaneous sumatriptan at a dose of $6 \mathrm{mg}$ is quickly absorbed, with a time to maximum plasma concentration of approximately 10 minutes and an average bioavailability of $96 \%$. Other routes of administration for sumatriptan, including oral, intranasal, and rectal, have a longer time to maximum plasma concentration, much closer to 1.5 hours, with bioavailability ranging from $14 \%$ to $19 \%{ }^{3}$

In two landmark studies, subcutaneous sumatriptan was shown to work better than placebo for the treatment of acute migraine. In 1991, Cady et al randomized otherwise healthy adult patients with migraine to subcutaneous sumatriptan $(n=734)$ or placebo $(n=370) .{ }^{10}$ These authors found that sumatriptan was more effective than placebo in reducing moderate or severe pain to mild or no pain ( $70 \%$ versus $22 \%$ ), completely relieving headaches (49\% versus 9\%), improving clinical disability (76\% versus 34\%), and reducing nausea and photophobia.

A second study in 1991 randomized 639 patients to $6 \mathrm{mg}$ or $8 \mathrm{mg}$ of subcutaneous sumatriptan or placebo. ${ }^{11}$ Those treated with sumatriptan had marked improvement in their headache at one hour and 2 hours as compared with those who received placebo. There was no significant difference noted between the $6 \mathrm{mg}$ and $8 \mathrm{mg}$ doses. Sumatriptan was also found to be more effective in reducing nausea, vomiting, photophobia, and phonophobia. Further, more participants treated with sumatriptan were able to function normally at one hour and 2 hours after administration of medication compared with placebo. In both of these early studies, patients who did not obtain relief from their initial dose of sumatriptan were given a second dose. The second dose of sumatriptan one hour after the first did not show any benefit in improving headache pain. These studies established that
$6 \mathrm{mg}$ of subcutaneous sumatriptan is an effective approach to treating migraine headache.

In 2000, Tfelt-Hansen et al summarized the results of 13 double-blind, randomized controlled trials in which subcutaneous sumatriptan $6 \mathrm{mg}$ had been compared with placebo. This review included 2108 patients treated with sumatriptan, $70 \%$ of whom had headache relief after one hour versus $19 \%$ of 1307 patients treated with placebo. Therefore, the therapeutic gain, ie, the percentage responding to active drug minus the percentage responding to placebo, was $51 \%$ $(70 \%-19 \%)$. In ten of these trials, the therapeutic gain was similar at 2 hours, indicating that the one-hour time frame was adequate for reassessment. ${ }^{3}$

In contrast, the therapeutic gain for $100 \mathrm{mg}$ of oral sumatriptan was found to be $32 \%$ two hours after administration in a review of 20 double-blind, placebo-controlled, randomized trials including 2928 patients treated with sumatriptan and 1653 patients treated with placebo. ${ }^{3}$

The efficacy of subcutaneous sumatriptan was also examined in a 2001 review of the use of triptans in Canada. The authors included only randomized placebo-controlled trials and calculated the therapeutic gain. They found that one hour after treatment subcutaneous sumatriptan clearly had the highest therapeutic gain $(50.98 \%$; 95\% confidence interval [CI] 47.7-54.21) compared with oral sumatriptan $100 \mathrm{mg}$, oral and wafer rizatriptan $10 \mathrm{mg}$, and oral zolmitriptan $2.5 \mathrm{mg}$. Two hours after administration, subcutaneous sumatriptan also had the highest therapeutic gain $(49.7 \%$; 95\% CI 43.8-55.7), but now closely followed by wafer rizatriptan $10 \mathrm{mg}$ (therapeutic gain 46.4\%; 95\% CI 37.3-55.5). A similar trend was observed for patients who were pain-free at one and two hours. Subcutaneous sumatriptan also had the highest therapeutic gain in clinical disability score of zero at one or 2 hours. The authors commented that subcutaneous sumatriptan may be the best option for patients who have migraine with rapid progression to severe intensity associated with high clinical disability. ${ }^{12}$

In a third systematic review of randomized controlled trials for acute migraine in 2002 by Oldman et al, subcutaneous sumatriptan showed similar results to the previous two reviews outlined. Other drugs included in this review were intranasal dihydroergotamine + sumatriptan, oral aspirin + metoclopramide, ergotamine + caffeine, paracetamol + aspirin, naratriptan, rizatriptan, sumatriptan, rapid-release tolfenamic acid, and zolmitriptan. Data on eletriptan from clinical trials by Pfizer Inc were also included. The authors calculated the number needed to treat as the reciprocal of the therapeutic gain, which can be 
interpreted as the number of people needed to treat in order to observe a positive outcome as compared with placebo. For headache relief, subcutaneous sumatriptan had the greatest efficacy at one hour after administration, with a number needed to treat of 2.1 (95\% CI 1.9-2.2) as compared with the next lowest number needed to treat, ie, intranasal dihydroergotamine $2 \mathrm{mg}(3.4 ; 95 \%$ CI 2.4-5.9). It also had the greatest efficacy at 2 hours and a number needed to treat of 2.0 (95\% CI 1.8-2.2). Multiple other drugs had numbers needed to treat in the upper 2-3 range and were statistically equivalent. For pain-free headache relief at 2 hours, subcutaneous sumatriptan clearly had the highest efficacy. For pain-free headache relief at 24 hours, oral eletriptan $80 \mathrm{mg}$ was superior (number needed to treat 2.8 ; 95\% CI 2.5-3.2) to subcutaneous sumatriptan (number needed to treat 3.2; 95\% CI 2.4-4.8). ${ }^{13}$

In 2009, the European Federation of Neurological Societies reviewed the available literature on clinical studies of migraine, migraine with aura, and migraine-like attacks. Based on their review of the literature and the expert opinion of the authors, they gave graded recommendations for drug treatment of pain from acute migraine headaches. Triptans were given a level A recommendation based on the previous literature. The authors recommended $6 \mathrm{mg}$ of subcutaneous sumatriptan or intravenous aspirin with or without metoclopramide as first-line agents in the emergency treatment of acute migraine. ${ }^{14}$

Additionally, practice guidelines from the US Headache Consortium recommend triptans as the initial choice for the treatment of pain from acute moderate to severe migraine headaches in patients who have no contraindications to these medications. This recommendation is based on grade A evidence, ie, multiple, randomized controlled trials with consistent results. The authors recommended that patients with nausea or vomiting be given intranasal or subcutaneous sumatriptan, and that triptans may be used as the initial treatment for pain from migraine headaches of any severity when other medication have previously failed to provide pain relief. Both of these are grade $\mathrm{C}$ (expert consensus) recommendations. ${ }^{15}$

Subcutaneous sumatriptan has been widely studied and found to be superior to placebo, and along with other triptans is recommended as first-line therapy for acute treatment of moderate to severe migraine. It has the fastest onset of action of any triptan, with effects seen as early as ten minutes, and has a high rate of improvement in pain and relief of pain at one hour. It is also a good option in patients suffering from migraine associated with nausea and vomiting.

\section{Comparative trials}

There have been few studies of subcutaneous sumatriptan using active comparators, but it has been compared with intravenous aspirin, oral eletriptan, metoclopramide, prochlorperazine, and trimethobenzamide-diphenhydramine.

In a 1999 study, the ASASUMAMIG Study Group compared intravenous acetylsalicylic acid lysinate (L-ASA, an intravenous form of aspirin) and subcutaneous sumatriptan. This was a randomized, double-blind, double-dummy, multicenter, placebo-controlled study including 279 migraine patients. Patients were asked to rate their headaches on a verbal rating scale, with the primary outcome of the study being headache relief. Secondary outcomes included headache relief defined by change in a $100 \mathrm{~mm}$ visual analog pain scale, number of patients who were pain-free, rate of recurrent headaches, changes in migraine-associated symptoms, time until the patient could return to work at normal levels, and other adverse events. ${ }^{16}$

The authors found subcutaneous sumatriptan to be superior to L-ASA, as measured by the primary outcome (91.2\% versus $73.9 \%, P=0.001)$. Both medications were superior to placebo for migraine relief. Sumatriptan was also superior to L-ASA for the secondary outcomes of change in visual analog score, proportion of patients who were pain-free at 2 hours, and time until the patient could return to work. L-ASA performed similarly to subcutaneous sumatriptan for some migraine-associated symptoms, such as nausea, vomiting, photophobia, phonophobia, and visual disturbances. Recurrent headaches 24 hours after treatment were similar for both groups (L-ASA $18.2 \%$, sumatriptan $23.1 \%$, placebo $20 \%)^{16}$

The rate of adverse events was higher for subcutaneous sumatriptan than for L-ASA $(32.8 \%$ versus $7.6 \%$, respectively, and $9.3 \%$ for placebo). The most common adverse events for subcutaneous sumatriptan were tiredness/ weariness/fatigue (12.9\%), dizziness/vertigo (6.8\%), and injection site reactions $(6.0 \%)$. Four participants had chest pain. Eleven adverse events were rated as "severe" without further description, with six of these events related to sumatriptan. ${ }^{16}$

In 2005, Schoenen et al compared oral eletriptan $80 \mathrm{mg}$ with subcutaneous sumatriptan $6 \mathrm{mg}$ in patients who had previously used subcutaneous sumatriptan in a randomized, open crossover trial. This was the first study directly comparing subcutaneous sumatriptan with an oral triptan and used patient preference for future treatment as the primary outcome. The authors found no significant difference in patient preference between the two drugs $(50.6 \%$ eletriptan 
versus $43 \%$ subcutaneous sumatriptan, no $P$ value given). Secondary outcomes included efficacy of the drugs. One hour after administration, subcutaneous sumatriptan was superior to eletriptan for both headache relief and number of patients who were pain-free. Two hours after administration, subcutaneous sumatriptan had a higher rate for headache relief $(87 \%$ subcutaneous sumatriptan versus $82 \%$ eletriptan, $P<0.05$ ) but the two drugs had similar percentages of patients who were pain-free (61\% sumatriptan versus $58 \%$ eletriptan, no $P$ value given). ${ }^{17}$

In a 2005 study, Friedman et al compared the efficacy of intravenous metoclopramide with that of subcutaneous sumatriptan in emergency department patients with a chief complaint of headache who met International Headache Society guidelines for migraine with or without aura. This randomized, double-blind, clinical trial had a primary outcome of change in pain intensity 2 hours after treatment as measured by an 11-point pain scale. Of note, the dosing of metoclopramide was four $20 \mathrm{mg}$ doses every 30 minutes as needed, and it was also given with diphenhydramine for the first and third doses. The authors found that both intravenous metoclopramide and subcutaneous sumatriptan relieved pain to a similar extent at 2 hours. In terms of secondary endpoints, more participants treated with intravenous metoclopramide were pain-free at 2 hours and participants from both groups had similar rates of headache improvement at 24 hours. The most common adverse reactions at 2 and 24 hours for both groups were weakness, dizziness, and drowsiness, with rates being comparable between the two medication groups. At one hour, the subcutaneous sumatriptan group did have a significantly higher rate of chest heaviness. There were no reports of chest pain. ${ }^{18}$

In 2006, Friedman et al compared subcutaneous sumatriptan with intramuscular trimethobenzamide-diphenhydramine, on the basis that intramuscular trimethobenzamidediphenhydramine would be superior to subcutaneous sumatriptan for relief of acute migraine.

The study was a randomized, double-blind, doubledummy design completed in the emergency department. The primary outcome was decreased pain as measured by an 11-point numerical rating scale at 2 hours, with secondary outcomes of a four-point pain scale and disability scale. Patients were also contacted at 24 hours by telephone for follow-up. The study was stopped after a preplanned interim analysis by the data monitoring committee because subcutaneous sumatriptan showed substantial benefit compared with intramuscular trimethobenzamide-diphenhydramine after enrollment of 40 patients. At 24-hour follow-up, the two groups had comparable pain relief. After discharge, significantly more patients in the subcutaneous sumatriptan group had nausea and some limitation to their usual daily activities. Both groups had similar rates of side effects and need for rescue medication. The authors concluded that subcutaneous sumatriptan was likely superior to trimethobenzamide-diphenhydramine, but that trimethobenzamide-diphenhydramine was effective and well tolerated, with a possible role in patients with contraindications to sumatriptan. ${ }^{19}$

Kostic et al compared subcutaneous sumatriptan with intravenous prochlorperazine in a 2010 randomized, doubleblind emergency department study. Sixty-eight patients who had a history of migraine and presented with a typical migraine headache were included. The primary outcome of the study was mean change in pain intensity 80 minutes after treatment, or at discharge if the patient was discharged prior to 80 minutes after medication administration. Secondary outcomes included changes in sedation and nausea reported by the patient. ${ }^{20}$

The authors found that both treatments were effective in reduction of headache, with the mean decrease in pain intensity being significantly higher for prochlorperazine than for sumatriptan. Changes in sedation and nausea were not significantly different between the groups. Twenty-eight percent of patients in the prochlorperazine group reported restlessness but did not require further treatment. No patient in the sumatriptan group reported chest pain. Proportions of recurrent headache were similar between the medication groups at 72 hours ( $43 \%$ intravenous prochlorperazine versus $63 \%$ subcutaneous sumatriptan, $95 \% \mathrm{CI}-31-60){ }^{20}$

In summary, there are few studies in which subcutaneous sumatriptan has been compared directly with other drugs. In those studies, the efficacy of subcutaneous sumatriptan was comparable with oral eletriptan and intravenous metoclopramide, was superior to intravenous aspirin and intramuscular trimethobenzamide-diphenhydramine, and was inferior only to intravenous prochlorperazine for pain relief.

\section{Primary headaches}

Primary headaches are headaches that are not secondary to some identified discrete pathology, such as meningitis or subarachnoid hemorrhage. These include migraine, probable migraine, tension, and cluster headaches. ${ }^{2}$ Very little is known about the differences in etiology and treatment of primary headaches. Primary headaches have been reported to account for a complaint of headache in the overwhelming majority of ambulatory patients. ${ }^{21}$ In the emergency department or clinic 
setting, it has been shown that clinicians can treat primary headaches with similar classes of medication, regardless of headache classification. ${ }^{22-24}$

Subcutaneous sumatriptan has been shown to work in primary headaches. ${ }^{4,5}$ In a 2007 study, Miner et al used subcutaneous sumatriptan as a first-line agent for benign, undifferentiated headaches in all patients presenting to the emergency department, regardless of migraine history. Type of headache at the time of presentation was classified as migraine, probable migraine, or tension headache based on International Headache Society criteria. All patients received subcutaneous sumatriptan $6 \mathrm{mg}$ once enrolled. The primary outcome of this study was pain relief among the various headache subtypes. A $100 \mathrm{~mm}$ visual analog scale was used 30 and 60 minutes after administration to determine pain relief, and patients were contacted 48 hours after treatment to assess for adverse events and recurrence of headache. Of the 167 patients enrolled in this study, 84 (57.1\%) had migraine headache, $45(30.7 \%)$ had probable migraine headache, and $18(12.2 \%)$ had tension headache. There was no difference in pain relief between the headache subtypes as measured by visual analog score, with 50/84 (60\%) of migraine patients, $25 / 45$ (56\%) of probable migraine, and 12/18 (67\%) experiencing at least a $50 \%$ reduction in visual analog score $(P=0.72) 60$ minutes after treatment. ${ }^{24}$

Those without a history of migraine headaches had a similar rate of good response to subcutaneous sumatriptan as compared with those with a history of migraine headaches. Thirty-six adverse events were recorded, including 13 patients with chest and neck burning or tightness. All of these patients had symptoms for less than 60 minutes and electrocardiograms were performed, with no abnormalities noted after these symptoms. ${ }^{24}$

Ninety-five $(64.6 \%)$ of patients were reached by telephone at 48 hours after enrollment. Sixty-three (66.3\%) had continued headache, with 26 rating their headache as "severe," six as "moderate," and 31 as "light". There was no difference between International Headache Society groups regarding continued symptoms. The authors concluded that subcutaneous sumatriptan is effective for the treatment of primary headaches in the emergency department, based on International Headache Society classification of headache, with similar rates of adverse events as reported previously. ${ }^{24}$

These findings are similar to those of a 2000 study by Lipton et al, where the authors randomized patients to placebo or oral sumatriptan in a 4:1 fashion in the clinic setting. All patients had a history of migraine, but were instructed to take the assigned medication for any headache, regardless of whether it was migraine or not. They used diaries to record pretreatment characteristics of their headaches, which were later classified by the study investigators using International Headache Society criteria. Two hundred and forty-nine patients were treated for 1576 headaches, 1110 migraine, 103 migrainous, and 363 tension-type headaches. Sumatriptan $50 \mathrm{mg}$ orally was superior to placebo in all three headache groups 4 hours after dosing. ${ }^{22}$

All of the patients had a history of migraine in the Lipton et al study, while patients in the Miner et al study did not require a previous diagnosis of migraine for inclusion. The majority of the patients in these studies had migraine or migrainous headache. There was a significant minority in both studies with tension-type headaches who had relief with sumatriptan. There were no patients in these studies with cluster headache. However, it is known that sumatriptan is effective in patients with cluster headache. ${ }^{1}$ Both these studies suggest that sumatriptan in any form is an option for the treatment of primary headache, provided it is used in the appropriate patient population.

\section{Safety}

Subcutaneous sumatriptan is safe when it is given to patients without known cerebrovascular disease, with rare occurrences of serious drug events. ${ }^{25-27}$ Sumatriptan has been extensively studied since it was introduced. In clinical trials through to December 1998, more than 88,000 migraine patients had treated over 300,000 migraines with sumatriptan, and 2000 normal healthy volunteers had also been given sumatriptan. In a report of marketing data in 1998, there were more than nine million patient exposures and over 236 million attacks treated with sumatriptan. ${ }^{25}$ Hall et al conducted a large study of 63,575 migraine patients and 77,239 controls with the objective of estimating incidence of stroke, cardiovascular events, or death in migraine patients and evaluating for an association between these events and those treated with triptans. Cardiovascular events were defined as myocardial infarction, transient ischemic attack, and ischemic heart disease. In this study, 13,664 patients were prescribed triptans, and the authors found the risk of these events to be no different to that at baseline in the population studied.

Of the different triptan types and formulations, subcutaneous sumatriptan appears to have the highest rate of side effects, most of which are mild to moderate and transient. Common side effects include injection site reactions and the so-called "triptan sensations". These include tingling, numbness, a sensation of warmth, and heaviness and pressure, 
principally in the chest and neck. Neurologic symptoms include somnolence and dizziness, although there is some debate over whether these are a consequence of the migraine process or an adverse event of the drug. ${ }^{25,28-30}$

Rates of adverse events in short-term clinical studies are variable, ranging from $<10 \%$ to $>50 \%{ }^{25,29,30}$ Injection site reactions are the most common side effect, reported in 59\% of patients who received subcutaneous sumatriptan versus $24 \%$ of those who received placebo in a summary of those participating in the short-term clinical trials. More than $80 \%$ of these adverse events were mild to moderate, with most lasting less than 3 hours. Tingling, dizziness, and warm/ hot sensations were reported by $<15 \%$ in the subcutaneous sumatriptan groups versus $<4 \%$ in the placebo groups. The remainder of adverse events reported, including burning sensation, feeling of heaviness or tightness, flushing, neck pain/stiffness, weakness, and numbness, accounted for less than $10 \%$ in the subcutaneous sumatriptan group and less than $4 \%$ in the placebo group. In long-term clinical trials lasting up to two years, the types of adverse events were similar to those in the short-term clinical trials. ${ }^{25}$ The frequency of adverse events was similar to or less than that in the short-term clinical trials. ${ }^{25,28}$ In total, $6 \%-12 \%$ of patients using subcutaneous sumatriptan withdrew from long-term clinical trials because of adverse events versus $7 \%$ and $4 \%$ of those using tablet and nasal spray forms of sumatriptan, respectively. ${ }^{25,28,30,31}$

The proportion of patients reporting chest symptoms, such as tightness and pressure, is higher in clinical practice than in the clinical trials, in the range of $20 \%-40 \%$ in clinical practice and $3 \%-5 \%$ in the clinical trials. The majority of patients experiencing these symptoms are young and middle-aged women, who in general have a lower body mass index and lower cardiovascular risk. Approximately 50\% of patients in the clinical trials also experienced feelings of tightness and heaviness in other areas of the body. However, the incidence of myocardial ischemia with these events has been rare. The overwhelming majority of these events have showed no causal relationship to myocardial ischemia by electrocardiogram, angiography, cardiac enzyme measurement, or temporal data. ${ }^{26,29,33,34}$

The incidence of serious cardiac and cerebrovascular events with subcutaneous sumatriptan is relatively rare. Sumatriptan is contraindicated in patients with coronary artery disease (CAD), cerebrovascular disease, arterial disease (eg, Raynaud's), or peripheral arterial disease due to reported events of myocardial infarction, life-threatening cardiac dysrhythmias, and death. It is recommended that sumatriptan is not given to patients in whom CAD status is unclear or to those with risk factors, such as hypertension, hypercholesterolemia, smoking, diabetes mellitus, or a strong family history of CAD. This is excluding patients who have these risk factors but have had no evidence of CAD, cerebrovascular disease, or peripheral arterial disease after cardiovascular evaluation. ${ }^{25,28,34}$

Postmarketing data represent a valuable source of information on adverse events. They provide large numbers of patient exposures to a drug in clinical practice, increasing the detection of rare events, and also provide ongoing information about the how the drug is tolerated in the population. However, the utility of the information is limited compared with data from a clinical trial. There are no control groups or follow-up of outcomes, there is a general lack of uniform definition of adverse events, and event reporting is left to the discretion of patients and/or caregivers. It is impossible to calculate the true incidence of adverse events for sumatriptan because we do not know the total number of adverse events or the total number of patients who use sumatriptan..$^{25,28}$

In a review of postmarketing data between 1992 and December 1998, there were 451 reports of serious cardiac adverse events within 24 hours of taking sumatriptan. These events include myocardial ischemia, angina/chest pain, increased cardiac enzymes, any ST segment change, serious dysrhythmias, cardiovascular test abnormalities, asystole, ventricular tachycardia or fibrillation, and vasospasm. Most patients with adverse cardiac events had risk factors for $\mathrm{CAD}$ or were known to have CAD. In the same review, 92 cardiovascular and 10 neurologic deaths were reported at any post-dose time of subcutaneous sumatriptan, tablet, or nasal spray in nine million patients for more than 236 migraine attacks. ${ }^{25}$ This indicates that in the clinical setting there is likely to be a cardiovascular risk from sumatriptan, and that it is small but likely higher in patients with known cardiovascular disease.

Vasoconstriction is mostly mediated by $5-\mathrm{HT}_{1 \mathrm{~B}}$ and $5-\mathrm{HT}_{2 \mathrm{~A}}$ receptors in human coronary arteries. ${ }^{35}$ Triptans are most selective for $5-\mathrm{HT}_{1 \mathrm{~B}}$ and $5-\mathrm{HT}_{1 \mathrm{D}}$ receptors. In the isolated or in vitro human coronary artery, $5-\mathrm{HT}_{2 \mathrm{~A}}$ receptors generally produce greater contraction than $5-\mathrm{HT}_{1 \mathrm{~B}}$ receptors. ${ }^{36-41}$

In a study of the cardiovascular effects of triptans, several triptans, including sumatriptan, showed small comparable contractions in isolated human coronary arteries from organ donor patients who were deceased from noncardiac etiologies. The authors found that the ratio between unbound maximum plasma concentrations $\left(\mathrm{C}_{\text {max }}\right)$ of triptans after administration 
of a clinically effective dose and the concentration of different compounds required to obtain $50 \%$ contraction of the coronary artery $\left(\mathrm{EC}_{50}\right)$ was low. This ratio provides a relationship between maximal plasma concentrations of a triptan and its vasoconstrictor effect. $\mathrm{A}_{\max } / \mathrm{EC}_{50}$ ratio of 1 would produce $50 \%$ maximum contraction, and all triptans were below 0.4 . This study would suggest that triptans are not expected to cause significant $\mathrm{CAD}$ and do not produce significant coronary artery contraction at the doses typically used to treat migraine headaches. ${ }^{40,42}$

In a more recent study, Edvinsson et al examined the $\mathrm{C}_{\max } / \mathrm{EC}_{50}$ ratio of several triptans, in addition to quantifying their vasoconstrictor effect in isolated human cerebral and coronary arteries and the number of 5- $\mathrm{HT}_{1 \mathrm{~B}}$ receptors in atherosclerotic and normal coronary arteries. This study found the triptans were three times more potent as vasoconstrictors in cerebral arteries than in coronary arteries. No difference was found in the expression of $5-\mathrm{HT}_{1 \mathrm{~B}}$ receptors in atherosclerotic and normal coronary arteries. The $\mathrm{C}_{\max } / \mathrm{EC}_{50}$ ratio for cerebral arteries approached 1 , while the $\mathrm{C}_{\max } / \mathrm{EC}_{50}$ ratio for coronary arteries was much lower, the highest being subcutaneous sumatriptan with a mean ratio of $0.11(0.08-0.57)$. These findings support the clinical observation that triptans are safe drugs with a minimal risk of adverse coronary events in the appropriate patient population. ${ }^{43}$

Building on the results of the above studies, multiple studies have shown a clinically insignificant decrease in mean coronary artery diameter when subcutaneous sumatriptan is given at therapeutic levels or supratherapeutic levels to individuals without migraine and without CAD. ${ }^{44} 48$ Additionally, the relevant study results are also consistent with those of a double-blind, crossover, positron emission tomography study in which images of the heart were interpreted before and 10 minutes after administration of subcutaneous sumatriptan or placebo in 19 patients. All patients were women, had a low risk of cardiovascular disease, and were younger than 62 years. Three women developed neck tightness and one developed chest tightness after receiving subcutaneous sumatriptan. Overall, no significant change in myocardial perfusion was observed, including in the patients with neck or chest tightness. ${ }^{49}$

It is not known whether sumatriptan has an effect on diseased coronary arteries. In one small study by Glaxo Wellcome in 1994, 16 patients with at least 50\% stenosis in at least one vessel segment were given subcutaneous sumatriptan $(n=11)$ or placebo $(n=5)$. There were no significant changes in mean coronary artery diameter between the treatment groups. ${ }^{25}$ The main limitation of this study was the small sample size. Given the contraindication of sumatriptan in patients with known CAD, this study is unlikely to be repeated. In a post hoc study of isolated human coronary arteries, the same authors examined the relationship between endothelial integrity in a coronary artery segment and the degree of contraction after administration of sumatriptan. They found that the degree of coronary vasoconstriction was inversely proportional to the integrity of the coronary endothelium, suggesting that sumatriptan has a more potent vasoconstrictive effect in healthy coronary arteries. ${ }^{50}$ Although the vasoconstrictor effect in the coronary arteries is widely accepted as clinically insignificant in patients with no CAD, it is possible that even a small constricting effect in those with pre-existing CAD could cause myocardial ischemia.

The Triptan Cardiovascular Safety Expert Panel published a consensus statement in 2004 regarding the cardiovascular safety profile of triptans in the acute treatment of migraine. The authors concluded that the chest symptoms associated with triptan sensations in placebo-controlled clinical trials, excluding those with significant cardiovascular risk factors, were generally "transient, mild, and nonserious". They also concluded that in long-term clinical trials which excluded participants with known ischemic heart disease, the chest symptoms reported after triptan use were not associated with serious cardiovascular outcomes. Their conclusion was that triptans have minimal effects on the coronary arteries at therapeutic plasma concentrations. ${ }^{34}$

Postmarketing surveillance data in the consensus statement were also addressed, with the caveat that one cannot depend on surveillance data alone to examine for a causal association between triptan use and serious cardiovascular adverse events. The authors stated that the rate of serious cardiovascular adverse events is very small given the widespread use of triptans. However, it was emphasized that while this risk is low, it cannot be ignored, because use of triptans can lead to serious adverse effects, including death. ${ }^{34}$

In conclusion, subcutaneous sumatriptan has the highest rate of side effects of the triptans, with most side effects lasting less than 3 hours and characterized as mild to moderate. Of the triptans available, subcutaneous sumatriptan has the fastest onset of action, but has also been associated with the most adverse events. However, in general, it is well tolerated by patients. There is a rare but well described risk of serious cardiovascular events associated with subcutaneous sumatriptan, as with all triptans in general. It is contraindicated in patients with known cerebrovascular disease, and clinicians must be vigilant in assessing for cerebrovascular 
risk factors in patients with unknown disease status prior to drug administration. It is important to consider the riskbenefit profile for every individual prior to giving subcutaneous sumatriptan, but for most patients sumatriptan is a safe approach for the treatment of migraine headache.

\section{Patient acceptance and compliance}

Subcutaneous sumatriptan has been shown to be effective for acute primary headache, with a rapid onset of action, and is a good option for those with nausea and vomiting. Side effects with this medication are generally mild to moderate and typically do not last long. However, of the triptan compounds and formulations, subcutaneous sumatriptan has the highest reported rate of side effects. Serious adverse events are rare in those for whom the drug is indicated. However, many patients dislike subcutaneous administration and "triptan sensations". Although patients value efficacy and onset of action in migraine treatment, other aspects of treatment are also important. Many newer migraine studies also examine patient satisfaction and preference as an endpoint, recognizing the value of a well rounded approach to migraine treatment.

In 1998, Dahlof and Saiers compared subcutaneous sumatriptan and oral sumatriptan use in a population of 707 Swedish patients with migraine, who in total had administration of over 76,000 subcutaneous sumatriptan injections, $56,000100 \mathrm{mg}$ tablets, and 20,000 $50 \mathrm{mg}$ tablets. The authors undertook a telephone survey of patients previously diagnosed at the clinic with migraine who had used subcutaneous or oral sumatriptan. Overall, oral sumatriptan was more frequently used than subcutaneous sumatriptan. Fifty-one percent of patients used sumatriptan $100 \mathrm{mg}$ most frequently, $22 \%$ used sumatriptan $50 \mathrm{mg}$ tablets most frequently, and $21 \%$ used sumatriptan injection most frequently. Oral sumatriptan was preferred to subcutaneous sumatriptan by the patients surveyed. Thirty-two percent of patients overall preferred oral sumatriptan $100 \mathrm{mg}, 15 \%$ preferred subcutaneous sumatriptan, and $46 \%$ did not state a preference. The most common reason for tablet preference was fewer side effects and ease of administration. Subcutaneous sumatriptan was rated by patients as the most effective dosing formulation. Thirty-eight percent said it was the most effective form as compared with $14 \%$ and $4 \%$ rating sumatriptan $100 \mathrm{mg}$ and $50 \mathrm{mg}$ tablets as most effective, respectively, with $45 \%$ not stating a preference. Of those who rated subcutaneous sumatriptan as most effective, efficacy and quick onset of action were the most common reasons noted for giving the top rating. Ninety-one percent stated efficacy was the main reason for preferring the injection. Of those who found the tablets to be most effective, the reasons given were fewer side effects and lack of experience with other dosing forms. Side effects were less frequent in those who used sumatriptan tablets. $^{51}$

Overall, 94\% of 602 patients who used sumatriptan during the study rated sumatriptan as better or much better than previous therapies used for migraine. Listed previous therapies did not include other triptans. Just under $15 \%$ of the 707 patients had stopped using sumatriptan. The most frequently cited reasons for no longer using sumatriptan were its short duration of effect, concern about side effects, and lack of efficacy in controlling migraine pain. A large percentage of patients did not have an overall drug preference. ${ }^{51}$

This study illustrates how different dosing forms of sumatriptan can be used by migraine patients. As the authors state in their discussion, the results suggest that subcutaneous sumatriptan can be used in patients whose primary concern with treatment is speed of onset and efficacy of pain relief rather than possible adverse events. Similarly, oral sumatriptan may be a better agent in patients who have experienced some adverse effects from subcutaneous sumatriptan and wish to avoid them, or who are more willing to risk inferior treatment efficacy in order to avoid adverse events. ${ }^{51}$

A retrospective study from 2004 examined reasons for patients switching triptans at a tertiary headache center. Participants included patients followed for migraine headaches for at least one year who were using a triptan for acute migraine management and had previously used at least one other triptan or different triptan formulation. ${ }^{52}$

Patients who used subcutaneous sumatriptan initially were significantly less likely to switch because of "incomplete or no relief" as compared with all other drugs studied, which included oral sumatriptan 25, 50, and $100 \mathrm{mg}$, sumatriptan nasal spray, zolmitriptan, rizatriptan, and naratriptan. Subcutaneous sumatriptan performed comparably or better than other drugs in other categories such as recurrence, time to relief, inconsistency, and "other triptan or formulation is better". Nearly $20 \%$ of patients who had initially used subcutaneous sumatriptan and switched to another triptan or formulation did return to using subcutaneous sumatriptan. Those who used subcutaneous sumatriptan first were more likely to switch because of side effects (39\% of patients) than those who first used sumatriptan $25 \mathrm{mg}$ and $50 \mathrm{mg}$, zolmitriptan, rizatriptan, and naratriptan.

This study highlights the variety of reasons patients switch triptans or drug formulations. Similar to previous findings, subcutaneous sumatriptan performed well compared 
with other triptans in area such as efficacy, time to relief, and headache recurrence, but had the highest percentage of patients switching due to adverse events. ${ }^{52}$

Similar findings were reported in a 2010 study in which 343 migraine patients answered a questionnaire regarding their patterns of triptan use and adverse events. The most frequently used triptan was sumatriptan and the most frequent triptan discontinued was subcutaneous sumatriptan. The majority of patients $(72 \%)$ continued to use triptans, while $28 \%$ discontinued use of triptans altogether. Of patients continuing to take triptans, $85 \%$ of those continued their use of the medication because of its efficacy. Most patients discontinued the use of triptans because of adverse events (59\%) and not because of drug ineffectiveness. ${ }^{32}$

A pilot study that looked at patient preference for smaller doses of subcutaneous sumatriptan compared $3 \mathrm{mg}$ versus $6 \mathrm{mg}$ of subcutaneous sumatriptan for the treatment of acute migraine headache. Thirty patients were enrolled in this open crossover study. Eighty percent of patients preferred the $3 \mathrm{mg}$ dose to the $6 \mathrm{mg}$ dose when asked, "After trying both doses, which dose do you prefer?" Although no statistical tests were applied, the proportions of pain-free responses were similar in both groups, with $57 \%$ of the $3 \mathrm{mg}$ patients reporting being pain-free at one hour versus $53 \%$ being painfree in the $6 \mathrm{mg}$ group. At 2 hours, $87 \%$ were pain-free in the $3 \mathrm{mg}$ group and $80 \%$ were pain-free in the $6 \mathrm{mg}$ group. More side effects were reported with the $6 \mathrm{mg}$ dose than with the $3 \mathrm{mg}$ dose. ${ }^{53}$

Recently introduced is a needle-free form of sumatriptan, whereby subcutaneous sumatriptan is injected through a small hole in the skin created by a ram and piston system under compressed nitrogen gas. This system has been shown to be bioequivalent to subcutaneous sumatriptan delivered with a needle-based system and can be used successfully by migraine patients. ${ }^{54,55}$ This may offer an additional route of administration for patients intolerant of subcutaneous dosing using a needle.

\section{Conclusion}

Sumatriptan, in both its oral and subcutaneous form, is an effective treatment for acute migraine headache, and can be used in patients with known migraine syndrome and in those with primary headaches when a secondary cause has been excluded. Subcutaneous sumatriptan and other triptans are recommended as a first-line treatment for moderate to severe acute migraine based on the existing literature and expert consensus. Subcutaneous sumatriptan may be most effectively used in patients with moderate to severe migraine headache associated with nausea and vomiting. It has also performed well versus other medications for treatment of migraine in limited comparative trials.

Sumatriptan is associated with a rare but well described risk of cardiovascular adverse events. For this reason, it should not be used in patients with known cerebrovascular disease. Its use should also be limited in patients with cardiovascular risk factors whose cerebrovascular disease status is unknown. Injection site reactions and triptan sensations are the most common side effects of subcutaneous sumatriptan. Further, subcutaneous sumatriptan has the highest rate of side effects of all the triptans and their formulations, and was reported to be at least $50 \%$ in one study.

In patient preference and compliance studies, patients who prefer subcutaneous sumatriptan do so because of its efficacy and speed of onset. The most common reason for discontinuation of subcutaneous sumatriptan is adverse drug reactions. The subcutaneous formulation appears to be more effective than the oral formulation, but the oral route appears to be better tolerated. Either formulation appears to be a good first-line agent for the treatment of acute migraine headache. It is important to consider the needs of the patient, their past medical history, and what aspects of migraine treatment are most important to the patient when considering treatment of acute migraine or primary headache.

\section{Disclosure}

The authors state there are no commercial, financial, or other relationships in any way related to this work that could create a potential conflict of interest.

\section{References}

1. Law S, Derry S, Moore RA. Triptans for acute cluster headache. Cochrane Database Syst Rev. 2010;4:008042.

2. Lipton RB, Bigal ME, Steiner TJ, Silberstein SD, Olesen J. Classification of primary headaches. Neurology. 2004;63:427-435.

3. Tfelt-Hansen P, De Vries P, Saxena PR. Triptans in migraine: A comparative review of pharmacology, pharmacokinetics, and efficacy. Drugs. 2000;60:1259-1287.

4. Shields KG, Goadsby PJ. Serotonin receptors modulate trigeminovascular responses in the ventroposteromedial nucleus of thalamus: a migraine target? Neurobiol Dis. 2006;23:491-501.

5. Sprenger T, Goadsby PJ. Migraine pathogenesis and state of pharmacological treatment options. BMC Med. 2009;7:71.

6. Hoskin K, Kaube H, Goadsby PJ. Sumatriptan can inhibit trigeminal afferents by an exclusively neural mechanism. Brain. 1996;119: 1419-1428.

7. Goadsby PJ. The pharmacology of headache. Prog Neurobiol. 2000;62: 509-525.

8. Levy D, Jakubowski M, Burstein R. Disruption of communication between peripheral and central trigeminovascular neurons mediates the antimigraine action of 5HT 1B/1D receptor agonists. Proc Natl Acad Sci U S A. 2004;101:4274-4279. 
9. Bartsch T, Knight YE, Goadsby PJ. Activation of 5-HT(1B/1D) receptor in the periaqueductal gray inhibits nociception. Ann Neurol. 2004:56:371-381.

10. Cady RK, Wendt JK, Kirchner JR, Sargent JD, Rothrock JF, Skaggs H. Treatment of acute migraine with subcutaneous sumatriptan. JAMA. 1991;265:2831-2835.

11. Treatment of migraine attacks with sumatriptan. The Subcutaneous Sumatriptan International Study Group. N Engl J Med. 1991;325: 316-321.

12. Gawel MJ, Worthington I, Maggisano A. A systematic review of the use of triptans in acute migraine. Can J Neurol Sci. 2001;28:30-41.

13. Oldman AD, Smith LA, McQuay HJ, Moore RA. Pharmacological treatments for acute migraine: quantitative systematic review. Pain. 2002;97:247-257.

14. Evers S, Afra J, Frese A, et al. EFNS guideline on the drug treatment of migraine - revised report of an EFNS task force. Eur $J$ Neurol. 2009; 16:968-981.

15. Silberstein SD. Practice parameter: Evidence-based guidelines for migraine headache (an evidence-based review). Report of the Quality Standards Subcommittee of the American Academy of Neurology. Neurology. 2000;55:754-763.

16. Diener HC. Efficacy and safety of intravenous acetylsalicylic acid lysinate compared with subcutaneous sumatriptan and parenteral placebo in the acute treatment of migraine. A double-blind, double-dummy, randomized, multicenter, parallel group study. Cephalalgia. 1999;19: 581-588.

17. Schoenen J, Pascual J, Rasmussen S, Sun W, Sikes C, Hettiarachchi J. Patient preference for eletriptan $80 \mathrm{mg}$ versus subcutaneous sumatriptan $6 \mathrm{mg}$ : results of a crossover study in patients who have recently used subcutaneous sumatriptan. Eur J Neurol. 2005;12:108-117.

18. Friedman BW, Corbo J, Lipton RB, et al. A trial of metoclopramide versus sumatriptan for the emergency department treatment of migraines. Neurology. 2005;64:463-468.

19. Friedman BW, Hochberg M, Esses D, et al. A clinical trial of trimethobenzamide/diphenhydramine versus sumatriptan for acute migraines. Headache. 2006;46:934-941.

20. Kostic MA, Gutierrez FJ, Rieg TS, Moore TS, Gendron RT. A prospective randomized trial of intravenous prochlorperazine versus subcutaneous sumatriptan in acute migraine therapy in the emergency department. Ann Emerg Med. 2010;56:1-6.

21. Morgenstern LB, Huber JC, Luna-Gonzales H, et al. Headache in the emergency department. Headache. 2001;41:537-541.

22. Lipton RB, Stewart WF, Cady R, et al. Sumatriptan for the range of headaches in migraine sufferers: results of the Spectrum study. Headache. 2000;40:783-791.

23. Trainor A, Miner J. Pain treatment and relief among patients with primary headache subtypes in the ED. Am J Emerg Med. 2008;26:1029-1034.

24. Miner JR, Smith SW, Moore J, Biros M. Sumatriptan for the treatment of undifferentiated primary headaches in the emergency department. Am J Emerg Med. 2007;25:60-64.

25. Welch KM, Mathew NT, Stone P, Rosamond W, Saiers J, Gutterman D. Tolerability of sumatriptan: clinical trials and post-marketing experience. Cephalalgia. 2000;20:687-695.

26. Hall GC, Brown MM, Mo J, MacRae KD. Triptans in migraine: the risks of stroke, cardiovascular disease, and death in practice. Neurology. 2004;62:563-568.

27. O'Quinn S, Davis RL, Gutterman DL, Pait GD, Fox AW. Prospective large-scale study of the tolerability of subcutaneous sumatriptan for acute treatment of migraine. Cephalalgia. 1999;19:223-231.

28. Nappi G, Sandrini G, Sances G. Tolerability of the triptans: clinical implications. Drug Saf. 2003;26:93-107.

29. Brown EG, Endersby CA, Smith RN, Talbot JC. The safety and tolerability of sumatriptan: an overview. Eur Neurol. 1991;18:33-37.

30. Gross ML, Kay J, Turner AM, Jewsbury J, Cleal AL. Long-term efficacy of subcutaneous sumatriptan using a novel self-injector. Headache. 1995;35:601-606.
31. Tansey MJ, Pilgrim AJ, Martin PM. Long-term experience with sumatriptan in the treatment of migraine. Eur Neurol. 1993;33:310-315.

32. Ferrari A, Spaccapelo L, Sternieri E. Pharmacoepidemiology of triptans in a headache centre. Cephalalgia. 2010;30:847-854.

33. Visser WH, Jaspers MN, de Vriend RH, Ferrari MD. Chest symptoms after sumatriptan: a two-year clinical practice review in 735 consecutive migraine patients. Cephalalgia. 1996;16:554-559.

34. Dodick D, Lipton RB, Martin V, Papademetriou V, et al. Consensus statement: cardiovascular safety profile of triptans (5HT agonists) in the acute treatment of migraine. Headache. 2004;44:414-425.

35. Nilsson T, Longmore J, Shaw D, et al. Characterisation of 5-HT receptors in human coronary arteries by molecular and pharmacological techniques. Eur J Pharm. 1999;372:49-56.

36. Bax WA, Renzenbrink GJ, Van Heuven-Nolsen D, Thijssen EJ, Bos E, Saxena PR. 5HT receptors mediating contractions of the isolated human coronary artery. Eur J Pharm. 1993;239:203-210.

37. Connor HE, Feniuk W, Humphrey PP. 5-Hydroxytryptamine contracts human coronary arteries predominantly via 5 -HT2 receptor activation. Eur J Pharm. 989;161:91-94.

38. Chester AH, Martin GR, Bodelsson M, et al. 5-hydroxytryptamine receptor profile in healthy and diseased human epicardial coronary arteries. Cardiovasc Res. 1990;24:932-937.

39. Cocks TM, Kemp BK, Pruneau D, Angus JA. Comparison of contractile responses to 5-hydroxytryptamine and sumatriptan in human isolated coronary artery: synergy with the thromboxane A2-receptor agonist, U46619. Br J Pharmacol. 1993;110:360-368.

40. Maassen VanDenBrink A, Reekers M, Bax WA, Ferrari MD, Saxena PR. Coronary side-effect potential of current and prospective antimigraine drugs. Circulation. 1998;98:25-30.

41. Toda N, Okamura T. Comparison of the response to 5-carboxamidotryptamine and serotonin in isolated human, monkey, and dog coronary arteries. J Pharmacol Exp Ther. 1990;253:676-682.

42. van den Broek RW, Maassen VanDenBrink A, de Vries R, et al. Pharmacological analysis of contractile effects of eletriptan and sumatriptan on human isolated blood vessels. Eur J Pharmacol. 2000;407:165-173.

43. Edvinsson L, Uddman E, Wackenfors A, Davenport A, Longmore J, Malmsjo M. Triptan-induced contractile (5-HT1B receptor) responses in human cerebral and coronary arteries: relationship to clinical effect. Clin Sci. 2005;109:335-342.

44. MacIntyre PD, Bhargava B, Hogg KJ, Gemmill JD, Hillis WS. Effect of subcutaneous sumatriptan, a selective 5HT1 receptor agonist, on the systemic, pulmonary, and coronary circulation. Circulation. 1993;87: 401-405.

45. Macintyre PD, Bhargava B, Hogg KJ, Gemmill JD, Hillis WS. The effect of i.v. sumatriptan, a selective 5HT1 receptor agonist, on central haemodynamics and the coronary circulation. Br J Clin Pharmacol. 1992;34:541-546.

46. Hood S, Birnie D, Swan L, et al. Effects of subcutaneous naratriptan on systemic and pulmonary haemodynamics and coronary artery diameter in humans. J Cardiovasc Pharmacol. 1999;34:89-94.

47. Muir DF, McCann GP, Swan L, Clark AL, Hillis WS. Hemodynamic and coronary effects of intravenous eletriptan, a 5-HT1B/1D receptor agonist. Clin Pharmacol Ther. 1999;66:85-90.

48. Goldstein JA, Massey KD, Kirby S, et al. Effect of high-dose intravenous eletriptan on coronary artery diameter. Cephalalgia. 2004;24: $515-521$.

49. Lewis PJ, Barrington SF, Marsden PK, Maisey MN, Lewis LD. A study of the effects of sumatriptan on myocardial perfusion in healthy female migraineurs using $13 \mathrm{NH} 3$ positron emission tomography. Neurology. 1997;48:1542-1550.

50. Maassen VanDenBrink A, Bax WA, Ramrattan NN, Ferrari MD, Saxena PR. Human isolated coronary artery contraction to sumatriptan: a post hoc analysis. Cephalalgia. 1999;19:651-654.

51. Dahlöf CG, Saiers J. Sumatriptan injection and tablets in clinical practice: Results of a survey of 707 migraineurs. Headache. 1998;38: 756-763. 
52. Sheftell FD, Feleppa M, Tepper SJ, Volcy M, Rapoport AM, Bigal ME. Patterns of use of triptans and reasons for switching them in a tertiary care migraine population. Headache. 2004;44:661-668.

53. Landy SH, McGinnis JE, McDonald SA. Pilot study evaluating preference for $3 \mathrm{mg}$ versus $6 \mathrm{mg}$ subcutaneous sumatriptan. Headache. 2005;45:346-349.
54. Brandes JL, Cady RK, Freitag FG, et al. Needle-free subcutaneous sumatriptan: bioequivalence and ease of use. Headache. 2009;49: 1435-1444.

55. Cady RK, Aurora SK, Brandes JL, et al. Satisfaction with and confidence in needle-free subcutaneous sumatriptan in patients currently treated with triptans. Headache. 2011;51:1202-1211.

\section{Publish your work in this journal}

Patient Preference and Adherence is an international, peer-reviewed, open access journal focusing on the growing importance of patient preference and adherence throughout the therapeutic continuum. Patient satisfaction, acceptability, quality of life, compliance, persistence and their role in developing new therapeutic modalities and compounds to optimize clinical outcomes for existing disease states are major areas of interest. This journal has been accepted for indexing on PubMed Central. The manuscript management system is completely online and includes a very quick and fair peer-review system. Visit http://www.dovepress.com/ testimonials.php to read real quotes from published authors.

Submit your manuscript here: http://www.dovepress.com/patient-preference-and-adherence-journal 